anales de psicología, 2012, vol. $28, \mathrm{n}^{\circ} 2$ (mayo), 558-566 http://dx.doi.org/10.6018/analesps.28.2.128711
(C) Copyright 2012: Servicio de Publicaciones de la Universidad de Murcia. Murcia (España) ISSN edición impresa: 0212-9728. ISSN edición web (http://revistas.um.es/analesps): 1695-2294

\title{
Adaptación familiar de niños y niñas adoptados internacionalmente
}

\author{
Yolanda Sánchez-Sandoval ${ }^{1 *}$, Esperanza León ${ }^{2}$ y Maite Román² \\ ${ }^{1}$ Universidad de Cádir (España) \\ ${ }^{2}$ Universidad de Sevilla (España)
}

\begin{abstract}
Resumen: Este trabajo analiza la adaptación inicial de un grupo de niños y niñas procedentes de adopciones internacionales, así como el posterior ajuste tras como media tres años de convivencia con su familia adoptiva. Participaron 258 niños y niñas y sus respectivas familias residentes en cuatro comunidades del estado español que habían realizado sus adopciones en el extranjero. Padres, madres e hijos fueron visitados y entrevistados en sus propios hogares. Los resultados muestran en general una valoración muy positiva por parte de las familias respecto a su adaptación inicial, a pesar de que buena parte de los niños llegaron con problemas de desarrollo (evaluados con la escala Battelle) leves o moderados (19\%) o graves $(43 \%)$. Tanto el nivel de desarrollo inicial, la adaptación inicial, como los problemas de comportamientos manifestados por los menores se relacionan también con las dinámicas establecidas en estas familias como media tres años después de la adopción. Este artículo aporta datos que nos permiten insistir en la importancia del trabajo previo con las familias que van a adoptar, pero también en la importancia del apoyo y seguimiento en el tiempo una vez que las adopciones se han realizado.

Palabras clave: familias adoptivas; adaptación; problemas de comporta-
\end{abstract} miento; dinámica familiar; ajuste psicológico.

\section{Introducción}

Este trabajo se presenta en el contexto de una realidad cada vez más extendida en España como es la adopción de niños y niñas procedentes de otros países. Las adopciones internacionales comienzan a tener un cierto peso en nuestra sociedad sólo desde 1995, cuando España ratificara el Convenio de la Haya, acuerdo internacional en materia de adopciones. Es de destacar que, teniendo en cuenta los datos aportados por la Dirección General de Política Social, de las Familias y de la Infancia, del Ministerio de Sanidad Política Social e Igualdad, entre los años 2005 y 2009 se han llegado a realizar 19705 adopciones internacionales en nuestro país. De hecho, ha sido España el país donde se ha realizado un mayor número de adopciones en la última década (Palacios y Amorós, 2006). Con este nuevo escenario se plantea la necesidad de conocer cómo es la evolución de estos niños y niñas en sus nuevas familias.

Los niños y niñas que se adoptan en nuestro país llegan a unas familias que han sido valoradas por profesionales, y en la mayoría de los casos formadas para responder a este reto. Parte de esta formación suele ir dirigida a la preparación de los padres respecto a la llegada de sus hijos, y a los momentos iniciales de la convivencia (León, Sánchez-Sandoval, Palacios y Román, 2010). Para algunos autores, estos momentos iniciales serán fundamentales para el futuro desarrollo individual y ajuste familiar (Brodzinsky, Smith, y Brodzinsky, 1998; Levy-Shiff, 2001; Levy-Shift, Goldschmidt, y Har-

* Dirección para correspondencia [Correspondence address]: YOlanda Sánchez-Sandoval. Dpto. Psicología. Facultad de Ciencias de la Educación. Universidad de Cádiz. Avda. República Saharaui, s/n. 11519 Puerto Real (Cádiz, España). E-mail: volanda.sanchez@uca.es
Title: Family adaptation of internationally adopted children. Abstract: This paper analyzes the initial adaptation of a group of internationally adopted children and their subsequent adjustment after an average of three years living with their adoptive families. The sample consisted of 258 children adopted internationally by families living in four different regional communities in Spain. Fathers, mothers and children were visited and interviewed in their own homes. Results show very positive evaluations from families about children's initial adaptation, although most of the children arrived with mild/moderate $(19 \%)$ or severe $(43 \%)$ developmental problems (assessment by Battelle scale). Level of development at arrival, initial adjustment, and behavioral problems are related to the dynamics established in these families three years, on average, after adoption. This paper provides data that allow us to emphasize the importance of preparation of families who are going to adopt, but also the importance of the support and follow-up after adoption.

Key words: adoptive families; adaptation; family dynamics; psychological adjustment; behavioral problems.

even, 1991; Levy-Shiff, Zoran, y Shulman, 1997; Quinton, Rushton, Dance y Mayes, 1998). Algunos planteamientos teóricos han coincidido en describir ciertas etapas del ciclo vital de las familias adoptivas (Levy-Shiff, Bar y Har Even, 1990). La etiqueta luna de miel suele utilizarse para hacer alusión precisamente a los primeros momentos de adaptación mutua entre los miembros de esta nueva familia tras la incorporación de los niños y niñas adoptados. Se considera una etapa crítica, cuya principal tarea es la integración familiar, y donde el riesgo de rupturas es mayor (Berástegui, 2003; Fuentes-Peláez, 2006).

A pesar de su importancia, la investigación en adopción se ha centrado más en evaluar la adaptación de estos niños pasado cierto tiempo, normalmente años, desde el inicio de su convivencia con la familia adoptiva que en el análisis de los momentos iniciales de la misma. Entre los estudios que analizan estos aspectos en nuestro país, los datos de Amorós (1987) muestran la existencia de problemas entre los adoptados, tanto al inicio de su convivencia con la nueva familia como posteriormente. Los problemas iniciales afectaban a áreas tan diversas como la salud, el desarrollo psicomotor, el lenguaje, la hiperactividad, el negativismo y la agresividad. Sin embargo, la frecuencia y seriedad de los problemas van disminuyendo de forma importante, aunque también es cierto que aparecen otros nuevos. Cuando ya llevan largo tiempo conviviendo con sus familias, el $40 \%$ de los que fueron adoptados siendo mayores de 2 años, frente al 20\% de los adoptados por debajo de esa edad, seguían manifestando diversos problemas. López y García (1997) analizan también la adaptación inicial de un grupo de niños en acogimiento preadoptivo en Murcia. Dos terceras partes de las familias del estudio indican que la adaptación no fue problemática, el resto sí encontró dificultades. Muestran que, en la adaptación 
inicial de niños y niñas de adopciones nacionales, el grupo de niños que evolucionó con dificultad presentaban un rendimiento intelectual, un desarrollo perceptivo y un desarrollo del lenguaje medio-bajo o con retardo. En los casos de adaptaciones más difíciles se habían adoptado niños como media mayores (7.12 años) en comparación con el grupo de adaptaciones sin dificultades (3.14 años). Otro estudio, también con adopciones nacionales en nuestro país (Molina, 2002), indica que las dificultades que los niños encuentran en el periodo de adaptación suelen presentarse desde el inicio y durante los seis meses siguientes de la convivencia; a partir del año apenas aparecen problemas nuevos en relación a esta adaptación. Van desapareciendo las dificultades relacionadas con la vinculación y adaptación mutua.

Respecto a adopciones internacionales, Fuentes-Peláez (2006) ha analizado la primera convivencia de las familias con sus hijos aún en el país de origen y antes de llegar a España. Buena parte de las familias $(80 \%)$ considera que el ajuste inicial en el país de origen es positivo, mientras que el resto lo vive con una fuerte presencia de dificultades. Casi todos $(96.7 \%)$ valoran aspectos que les satisfacen de sus hijos y que sienten que les facilita el ajuste inicial. Entre estas valoraciones destacan los deseos expresados por los niños y niñas de tener padre y madre (66.7\%), el estado emocional positivo y vital (50\%), y la facilidad de comunicación $(23.3 \%)$. También se identificaron aspectos difíciles (un $76.7 \%$ ), entre ellos los comportamientos externalizados (40\%) y los estados de ansiedad de los niños y niñas (20\%) representan la mayor fuente de estrés. Esta autora muestra que, tras un periodo como media de unos 18 meses de convivencia, la adaptación familiar es muy positiva y con altos niveles de satisfacción parental (Fuentes-Peláez, 2009). En el estudio de Berástegui (2007) el 58.1\% de los niños presenta una conducta más o menos normalizada al llegar a su hogar adoptivo, un $38.7 \%$ presentan un volumen de problemas de rango clínico, y un 3.2\% límite. Son los problemas sociales y los de atención, los que con mayor frecuencia al inicio presentaban un rango clínico o límite. Los datos muestran una mejora significativa en la mayoría de las áreas de comportamiento analizadas a lo largo del tiempo. Un reciente estudio realizado en el País Vasco (Loizaga, Louzao, de Aranzabal y Labayru, 2009) con 434 familias adoptivas pone de manifiesto la buena adaptación a su nueva familia realizada por los niños al llegar de sus países de origen (sólo un $8.2 \%$ presentó dificultad), y cómo durante el primer año de convivencia se consiguieron notables avances.

Aunque la adaptación de los niños a la familia pudiera ser evaluada desde diferentes dimensiones, priman los estudios centrados en evaluar el ajuste individual de los niños y niñas adoptados. Este ajuste psicosocial puede evaluarse teniendo en cuenta muy diferentes dimensiones. En este sentido, la manifestación de problemas de comportamiento entre niños y niñas adoptados es de los tópicos más analizados en las investigaciones con muestras adoptivas. La creencia de un mayor riesgo de los adoptados para desarrollar problemas de ajuste psicológico (Kirschner, 1990) ha dado lugar a in- numerables investigaciones, investigaciones preocupadas en su mayoría por la comparación entre adoptados y no adoptados. Juffer y van Ijzendoorn (2005) han realizado un metanálisis de trabajos relacionados con los problemas de comportamiento manifestados por niños y niñas adoptados internacionalmente. Concluyen los autores que la mayoría de los niños y niñas de adopción internacional presentan un buen ajuste, aunque acuden a servicios de salud mental con mayor frecuencia que los chicos no adoptados de los grupos control; sin embargo, los de adopción internacional presentan menos problemas de comportamiento y utilizan menos servicios de salud mental que los de adopciones nacionales.

Más allá de las dificultades comportamentales, distintas investigaciones coinciden en destacar que en el desarrollo de los niños y niñas procedentes de adopciones internacionales suelen diagnosticarse problemas de salud, retrasos en el crecimiento, y otros déficits emocionales, sociales y cognitivos (Hernández-Muela, Mulas, Téllez de Meneses, y Roselló, 2003; Johnson et al., 1992; O'Connor et al., 2000; Palacios, Sánchez-Sandoval y León, 2005a). Aunque es necesario precisar que no todos los niños y niñas de adopción internacional llegan a sus nuevos hogares con un marcado desajuste psicológico (Rutter, Kreppner y O’Connor, 2001; SánchezSandoval, Palacios y León, 2004), sino que es posible también encontrar niños y niñas con un desarrollo ajustado a su edad a pesar de las posibles carencias, separaciones y experiencias previas a la adopción.

Sin embargo, entendemos que para poder dibujar un perfil más completo de la adaptación familiar es necesario ir más allá de medidas individuales centradas en el niño o niña adoptados e incorporar medidas que evalúen otros aspectos del sistema familiar. Por ejemplo, en el estudio de Berástegui (2007) dentro de las dinámicas familiares, se destaca el papel de las expectativas como modulador de la relación estrésadaptación; de manera que las familias con una visión más realista de la adopción, capaces de anticipar las dificultades y de afrontarlas, tenían mejores niveles de adaptación. También Fuentes-Peláez (2009) ha mostrado la importancia que en el proceso de integración en las adopciones internacionales tienen la percepción de las familias sobre las necesidades de los niños, la propia capacidad de afrontamiento, así como las relaciones familiares. En cuanto a este último aspecto, diferentes investigaciones han puesto de manifiesto la buena salud de la que gozan las relaciones familiares en familias adoptivas (Bernedo, Fuentes, Fernández-Molina, y Bersabé, 2007; Lanz, Lafrate, Rosnati y Scabini, 1999; Levy-Shift, Zoran y Shulman, 1997; Palacios y Sánchez-Sandoval, 1996; 1998). Rueter y Koerner (2008), por ejemplo, compararon los patrones de comunicación entre familias adoptivas y no adoptivas usando medidas observacionales de afecto, apoyo y control parental, sin encontrar diferencias entre ambos grupos de familias. Rosnati y Marta (1997) sí muestran diferencias, los adoptados evaluaban de manera más positiva la comunicación con sus padres y percibían un mayor apoyo de ellos que los no adoptados. Estudios desarrollados en nuestro país, como el de Bernedo, Fuentes y Fernández (2005), 
nos muestran que las familias adoptivas perciben menor grado de conflicto con sus hijos que las familias no adoptivas. De todas formas, no abundan precisamente las investigaciones que analicen las interacciones y la dinámica de las familias adoptivas, a pesar de la valiosa información que estos aspectos pudieran aportar sobre la adaptación de niños y niñas adoptados. En este sentido, Arranz, Oliva, Olabarrieta y Antolín (2010), tras comparar familias con distintas estructuras en nuestro país, muestran que las adoptivas destacan por presentar los contextos más estimulantes (evaluados con la escala HOME), así como por mostrar, junto a las familias homoparentales, los estilos educativos más democráticos, menos permisivos y autoritarios. Son estos los estilos más favorables para el ajuste y el desarrollo infantil.

A pesar de que durante décadas se ha llegado a crear un importante corpus de investigación respecto a las sólidas relaciones entre las interacciones familiares y el ajuste y desarrollo infantil, poco es sabido en este sentido respecto a las familias adoptivas. Los resultados de Fuentes, Motrico y Bersabé (2003) muestran que las familias adoptivas que tienen menos conflictos con sus hijos son familias que les expresan afecto y son inductivos cuando tienen que poner normas y exigir su cumplimiento. Otros estudios con adolescentes adoptados han mostrado que los chicos que crecían en familias con importantes patrones comunicativos tenían menor riesgo de desarrollar problemas de ajuste (Rueter y Koerner, 2008). También se ha comprobado, en una muestra de niños adoptados procedentes de orfanatos rumanos, que los niños y niñas con un apego inseguro tenían más problemas de comportamiento según la opinión de los propios padres (Marcovitch et al., 1997). Estudios de adopción nacional han puesto de manifiesto que las relaciones familiares se vuelven más complicadas en caso de que los niños y niñas manifiesten mayores problemas de comportamiento, mostrando éstos, por ejemplo, un mayor distanciamiento emocional hacia sus padres (Palacios y Sánchez-Sandoval, 1996; Sánchez-Sandoval, 2002). Pero, como decíamos, hay una gran escasez de investigaciones que aporten datos, en primer lugar, respecto a las interacciones y dinámicas en familias adoptivas, y que, en segundo lugar, ayuden a entender la direccionalidad de las relaciones entre dinámicas familiares y ajuste personal de los adoptados. Por otro lado, los estudios se han centrado más en el análisis de las relaciones entre estas variables en el periodo adolescente que con niños adoptados más pequeños.

El objetivo de este trabajo es explorar, en una muestra de niños y niñas adoptados en otros países, cómo ha sido su adaptación. Estamos interesados en identificar tanto posibles variables relacionadas con la adaptación inicial de los chicos y chicas a sus familias adoptivas como con las posteriores relaciones familiares tras un periodo de convivencia en las familias. Igualmente, nos interesa conocer tanto las dificultades individuales relacionadas con su desarrollo como con su comportamiento. Nuestra hipótesis de partida es que la adaptación familiar, tanto la inicial como la actual, habrá sido más complicada cuando los niños y niñas adoptados presen- taban mayores dificultades en su desarrollo y en su comportamiento.

\section{Método}

\section{Participantes}

En este estudio participan 258 niños y niñas adoptados y sus familias adoptivas. La selección de la muestra se realizó teniendo en cuenta la representación que sobre el total de adopciones internacionales realizadas en nuestro país tenían los siguientes criterios: país de procedencia, comunidad de residencia, edad a la llegada y edad en el momento del estudio (transcurridos 3 años como media desde el inicio de la adopción). Los niños y niñas procedían de China (24.8\%), Colombia (19.4\%), Federación Rusa (16.3\%), Guatemala (9.3\%), India (17.1\%) y Rumanía (13.2\%). Fueron adoptados por familias residentes en las comunidades de Madrid, Valencia, Andalucía y Castilla y León. Un 36.4\% eran niños y un $63.6 \%$ niñas, fueron adoptados con 35.51 meses como media $(D T=30.214)$ y en el momento del estudio tenían una media de 74.21 meses $(D T=34.519)$.

\section{Instrumentos}

- Escala de desarrollo Battelle (Newborg, Stock y Wnek, 1988). Se tomaron dos medidas: la primera referente al nivel de desarrollo psicológico de los niños y niñas a la llegada a la familia adoptiva, a través de información retrospectiva de los padres, y, la segunda, respecto al desarrollo en el momento del estudio evaluando en el hogar al propio niño o niña. Los niños pueden ser clasificados como con retraso severo del desarrollo, moderado o sin retraso.

- Test de inteligencia WISC-r (Weschler, 1974), respecto a los niños y niñas que en el momento del estudio tenían a partir de 6 años.

- Información sociodemográfica e historia previa. En una entrevista diseñada para este estudio (Sánchez-Sandoval, Palacios y León, 2002), se recogió información sociodemográfica de la familia y características de la adopción (país, edades, emplazamientos previos, historia de institucionalización y calidad, existencia de malos tratos previos). - Adaptación inicial de los niños y niñas a la familia. A través de varias preguntas de la misma entrevista anterior se medía la adaptación inicial de los niños y niñas a varios aspectos de la vida familiar (de 1 a 5 , siendo 1 "muy insatisfactoria" y 5 "muy satisfactoria").

- Revised Rutter Parent Scale (RRPS) (Hogg et al., 1997) aporta una puntuación total de manifestación de problemas (incluye cuatro áreas: problemas de comportamiento, emocionales, de hiperactividad y en el comportamiento prosocial). En los items se van presentando distintos comportamientos (por ejemplo, "Muy inquieto, le cuesta quedarse sentado durante mucho tiempo"), y los padres deben evaluar la frecuencia de aparición (de 0 a 2 ) en sus hijos. Puntuaciones superiores indican mayor manifestación de pro- 
blemas. Al existir dos versiones distintas de la escala para edades preescolares y escolares, hemos recodificado las puntuaciones directas en valores tipificados (puntuaciones 2).

- Parent Report of Child Behavior to the Parent (Informe de los Padres sobre la Conducta del Niño, IPCN) de Schaefer y Edgerton (1977). Contiene cinco subescalas (implicación afectiva, distanciamiento afectivo, independencia, resistencia al control y obediencia), de cinco items cada una (por ejemplo, "Intenta hacer las cosas por sí mismo"). Para cada uno de los ítems, los padres señalarán en qué medida las características que se presentan son semejantes a las de sus hijos (en absoluto parecido (1), poco parecido (2), bastante parecido (3), completamente parecido (4)). Para este trabajo el coeficiente de fiabilidad alpha de Cronbach fue respectivamente en cada una de las subescalas de $.65, .64$, .66, .79 y .77 .

- Escala de Evaluación de Estilos Educativos (4er) (Palacios y Sánchez-Sandoval, 2000; Sánchez-Sandoval y Palacios, 2004). Mide, a través de 20 items en una escala Likert de 1 a 5 , la postura de los padres y madres a las dimensiones tradicionales de estilos educativos: afecto y comunicación, exigencias y control (por ejemplo, "Trato de no exigir mucho a mi hijo para no agobiarle"). El coeficiente de fiabilidad alpha de Cronbach fue de .73 en este estudio.

- Family Adaptability and Cohesion Evaluation Scales II (FACES II) (Olson, Portner y Bell, 1982) que evalúa dos dimensiones de la dinámica familiar: adaptabilidad (flexibilidad y respuesta al cambio de la estructura familiar) y cohesión (grado en que los miembros de una familia están separados o conectados entre sî). Padres y madres elijen en una escala Likert (1 a 5) la frecuencia con que la situación planteada ocurre en su familia (mayor frecuencia va asociada a puntuaciones superiores). Un ejemplo de item es el siguiente, "En mi familia es difícil cambiar las normas". En este trabajo el coeficiente de fiabilidad alpha de Cronbach fue de .82 .

\section{Procedimiento}

Las familias seleccionadas recibieron una carta de la correspondiente Administración Pública de su Comunidad Autónoma competente en materia de adopciones internacionales, exponiendo los objetivos del estudio y solicitando su participación. Una vez aceptaban, las familias recibían una llamada del equipo investigador concertando una cita. Una pareja de investigadores previamente entrenados visitaban a cada familia en sus respectivos hogares. Ya en el domicilio, uno de ellos se centraba en la evaluación del niño o la niña y el otro procedía a entrevistar a los padres. En la mayoría de los casos, la información se recogía en una única visita de unas tres horas de duración. Si se necesitaba más tiempo, se realizaba una segunda visita a la familia para completar la recogida de datos.

\section{Resultados}

\section{Adaptación inicial y desarrollo infantil a la llegada}

Las familias hacen una valoración muy positiva respecto a la adaptación inicial de los niños y niñas adoptados a distintos aspectos de la vida familiar. En la Tabla 1 se muestran los descriptivos. En una escala de 1 a 5 , en la que 5 es el polo más positivo, las medias son superiores a 4 en las distintas variables estudiadas (adaptación a la familia, y en concreto al padre, la madre, los hermanos, la familia extensa, horarios, comidas, escuela).

Tabla 1. Descriptivos de las dimensiones de adaptación inicial.

\begin{tabular}{|c|c|c|c|c|c|}
\hline & $N$ & Mínimo & Máximo & Media & $D T$ \\
\hline $\begin{array}{l}\text { Adaptación familiar } \\
\text { inicial }\end{array}$ & 257 & 2.40 & 5.00 & 4.6908 & .47400 \\
\hline Adaptación general & 239 & 2.00 & 5.00 & 4.7573 & .51826 \\
\hline Adaptación padre & 232 & 2.00 & 5.00 & 4.6250 & .72785 \\
\hline Adaptación madre & 253 & 1.00 & 5.00 & 4.6640 & .78791 \\
\hline $\begin{array}{l}\text { Adaptación } \\
\text { hermanos }\end{array}$ & 85 & 2.00 & 5.00 & 4.5647 & .85143 \\
\hline $\begin{array}{l}\text { Adaptación familia } \\
\text { extensa }\end{array}$ & 253 & 2.00 & 5.00 & 4.7391 & .55913 \\
\hline $\begin{array}{l}\text { Adaptación } \\
\text { horarios }\end{array}$ & 256 & 1.00 & 5.00 & 4.4531 & .88437 \\
\hline $\begin{array}{l}\text { Adaptación } \\
\text { comidas }\end{array}$ & 256 & 1.00 & 5.00 & 4.1758 & 1.1497 \\
\hline $\begin{array}{l}\text { Adaptación rendi- } \\
\text { miento académico }\end{array}$ & 210 & 1.00 & 5.00 & 4.1190 & 1.1575 \\
\hline $\begin{array}{l}\text { Motivación por } \\
\text { escuela }\end{array}$ & 219 & 1.00 & 5.00 & 4.4566 & .87874 \\
\hline $\begin{array}{l}\text { Integración } \\
\text { compañeros }\end{array}$ & 219 & 1.00 & 5.00 & 4.5479 & .80208 \\
\hline
\end{tabular}

Los datos aportados por la escala Battelle nos permiten diferenciar entre tres grupos de niños y niñas en el momento de su llegada a la familia adoptiva: aquellos con un desarrollo ajustado a su edad (38\% de la muestra), niños y niñas con problemas de desarrollo leves o moderados $(19 \%)$ y niños y niñas con problemas graves $(43 \%)$.

Hemos realizado ANOVAs para conocer si la adaptación inicial de estos niños y niñas fue diferente en relación con su nivel de desarrollo psicológico a su llegada a nuestro país evaluado con la escala Battelle. Estas comparaciones no muestran relaciones significativas $(\mathrm{p}>.05)$ entre el nivel de desarrollo psicológico inicial y la adaptación inicial familiar en general, así como la adaptación inicial al padre, a la madre, a los hermanos, a la familia extensa, a los horarios, a las comidas, a las costumbres o a la escuela. La única relación significativa de la adaptación inicial es con el rendimiento académico $(F(2,104)=6.183, p=.002)$, en el sentido de que dicha adaptación fue más negativa cuanto peor era el desarrollo psicológico inicial. 


\section{Dinámica familiar y comportamiento infantil tras un periodo de convivencia}

Se han analizado las correlaciones existentes entre las medidas que evalúan la dinámica familiar (cohesión y adaptabilidad, estilos educativos y comportamiento de los niños hacia sus padres) tras un periodo de como media tres años de convivencia en las familias adoptivas. Como se muestra en la Tabla 2, existen correlaciones significativas entre la manifestación de problemas en el momento del estudio y algunas de las dimensiones con la que se ha medido la dinámica familiar. Así, encontramos correlaciones negativas entre la manifestación de problemas y la dimensión afecto y comuni- cación $(\mathrm{r}=-.352, p<.01)$, también con la dimensión de control ( $\mathrm{r}=-.200, p<.01)$, en la escala de evaluación de estilos educativos de los padres (4er). También se hallaron correlaciones negativas con la dimensión de adaptabilidad ( $\mathrm{r}=-.206$, $p<.01)$, dentro de la escala de evaluación de la dinámica familiar (FACES II), o con la independencia ( $\mathrm{r}=-.262$, $p<.001$ ), mostrada por los niños y niñas hacia sus padres (IPCN). En esta última escala, son positivas las correlaciones encontradas entre la resistencia al control $(\mathrm{r}=.386, p<.01)$, y el distanciamiento afectivo $(\mathrm{r}=.325, p<.01)$, respecto a la manifestación de problemas en los niños y niñas en el momento del estudio.

Tabla 2. Correlaciones entre las dimensiones de dinámica familiar y problemas de comportamiento.

\begin{tabular}{|c|c|c|c|c|c|c|c|c|}
\hline & $\begin{array}{l}\text { Problemas } \\
\text { comportamiento }\end{array}$ & $\begin{array}{l}\text { Cohesión } \\
\text { (FACES) }\end{array}$ & $\begin{array}{l}\text { Adaptabilidad } \\
\text { (FACES) }\end{array}$ & $\begin{array}{l}\text { Afecto y } \\
\text { comunicación } \\
\text { (4er) }\end{array}$ & $\begin{array}{l}\text { Exigencias } \\
\text { (4er) }\end{array}$ & $\begin{array}{l}\text { Control } \\
\text { (IPCN) }\end{array}$ & $\begin{array}{l}\text { Obediencia } \\
\text { (IPCN) }\end{array}$ & $\begin{array}{l}\text { Distanciamiento } \\
\text { afectivo (IPCN) }\end{array}$ \\
\hline Problemas comportamiento & 1 & & & & & & & \\
\hline Cohesión (FACES) & -129 & 1 & & & & & & \\
\hline Adaptabilidad (FACES) & $-.206(* *)$ & $.594(* * *)$ & 1 & & & & & \\
\hline Afecto y comunicación (4er) & $-.352(* * *)$ & $.320(* * *)$ & $.242(* * *)$ & 1 & & & & \\
\hline Exigencias (4er) & -.143 & .012 & .064 & $.281(* * *)$ & 1 & & & \\
\hline Control (IPCN) & $-.200(* *)$ & .026 & $.184\left(^{*}\right)$ & $.202(* *)$ & $-.168(*)$ & 1 & & \\
\hline Obediencia (IPCN) & $-.503(* * *)$ & .089 & $.233(* *)$ & $.281(* * *)$ & .019 & .095 & 1 & \\
\hline Distanciamiento afectivo (IPCN) & $.325(* * *)$ & -.107 & -.135 & $-.329(* * *)$ & -.061 & $-.193(*)$ & $-.209(* * *)$ & 1 \\
\hline
\end{tabular}

\section{Análisis de clusters}

Teniendo en cuenta las anteriores relaciones, realizamos un análisis de conglomerados de $\mathrm{K}$ medias, con el objetivo de formar clusters de familias en función de sus puntuaciones en las medidas de dinámica familiar y comportamiento infantil en el momento del estudio. Así, se tuvieron en cuenta las siguientes variables: problemas de comportamiento de los niños y niñas en el momento del estudio, las tres escalas de estilos educativos (afecto y comunicación, exigencias y control) de 4er, las dos de adaptabilidad y cohesión de FACES, y las de distanciamiento afectivo y obediencia de IPCN.

Se seleccionaron los sujetos que no tenían valores perdidos en ninguna de las variables implicadas. Así, finalmente 154 participaron en este análisis. Para identificar posibles diferencias entre los sujetos que incluimos en este análisis y los que quedaron fuera hicimos un análisis de casos perdidos. Respecto a los datos más sociodemográficos, los análisis indican que son semejantes ( $p>$.05) en cuanto a la distribución por género, por país de procedencia, por estructura familiar, respecto al número de emplazamientos previos a la adopción, a la experiencia de institucionalización previa y a la calidad de la misma, así como respecto a la edad de llegada como la edad en el momento del estudio. Las diferencias muestran que entre los que participaron en este análisis existía una mayor proporción de niños que experimentaron malos tratos previos a la adopción $\left(\chi^{2}(1,154)=4.685, p<\right.$ $.05)$. Respecto a las variables de contenidos, no existen entre los dos grupos diferencias $(p>.05)$ en las dimensiones de dinámica familiar, es decir FACES, 4er ni IPCN. También se distribuyen de manera similar respecto al desarrollo psicológico evaluado con Battelle retrospectivo $(p>.05)$.

Se formaron tres conglomerados, compuestos por 13, 63 y 78 familias respectivamente. En la Tabla 3 se muestran los centros de los conglomerados finales.

Tabla 3. Centros de los conglomerados finales.

\begin{tabular}{llcl}
\hline & \multicolumn{3}{c}{ Conglomerado } \\
\hline & 1 & 2 & 3 \\
Afecto y comunicación (4er) & 3.90 & 4.16 & 4.48 \\
Exigencias (4er) & 3.83 & 4.01 & 4.00 \\
Control (4er) & 3.04 & 3.35 & 3.80 \\
Cohesión (FACES) & 4.27 & 4.31 & 4.43 \\
Adaptabilidad (FACES) & 3.83 & 3.97 & 4.11 \\
Obediencia (IPCN) & 2.09 & 2.69 & 3.20 \\
Distanciamiento afectivo (IPCN) & 2.00 & 1.51 & 1.20 \\
Problemas comportamiento (Rutter) & 1.89 & 0.40 & -0.82 \\
\hline
\end{tabular}

Tomando como referencia las puntuaciones de los centros de los conglomerados finales, así como los Análisis de la Varianza de cada dimensión en cada cluster (Tabla 4), denominamos a dichos clusters como Familias $A(\mathrm{n}=13)$, Familias $B(\mathrm{n}=63)$ y Familias $C(\mathrm{n}=78)$. Como muestran los análisis, las diferencias entre los grupos son significativas excepto en lo relativo a las escalas de Cohesión (FACES) y de Exigencias (4er). El tercer grupo, Familias $C$, es el que presenta menores problemas de comportamiento entre los niños y niñas adoptados por estas familias y una dinámica familiar más positiva, en el momento del estudio, entendida ésta como una dinámica familiar caracterizada por una mayor adaptabilidad, menor distanciamiento emocional por parte de los niños y niñas y mayor afecto y comunicación por parte de los padres, así como niveles altos de exigencias y control. El 
primer grupo, Familias $A$, se caracteriza por mayores problemas de comportamiento por parte de los niños y niñas y peor dinámica familiar. El segundo cluster, Familias B, muestra valores intermedios.

Tabla 4. ANOVA de los clusters por las dimensiones que participaron en su composición.

\begin{tabular}{llcc}
\hline & Gl & $F$ & $p$ \\
\hline Afecto y comunicación (4er) & 2.151 & 17.702 & .000 \\
Exigencias (4er) & 2.151 & 0.710 & .493 \\
Control (4er) & 2.151 & 13.357 & .000 \\
Cohesión (FACES) & 2.151 & 2.605 & .077 \\
Adaptabilidad (FACES) & 2.151 & 6.830 & .001 \\
Obediencia (IPCN) & 2.151 & 43.141 & .000 \\
Distanciamiento afectivo (IPCN) & 2.151 & 22.983 & .000 \\
Problemas comportamiento (Rutter) & 2.151 & 213.958 & .000 \\
\hline
\end{tabular}

\section{Descripción sociodemográfica de los clusters}

No hay diferencias significativas por género entre clusters $(p>.05)$. Existen diferencias significativas entre las edades de los niños y niñas de los diferentes grupos $(p<$ .05). Las pruebas posthoc, además, nos muestran que las Familias $A$ adoptaron niños y niñas mayores que las Familias $C$ (Tabla 5).

Tabla 5. Edad media en meses de los niños y niñas de cada cluster.

\begin{tabular}{lllll}
\hline & Familias A & Familias B & Familias C & F \\
\hline $\begin{array}{l}\text { Edad de los } \\
\text { niños y niñas a } \\
\text { la llegada }\end{array}$ & 55.23 & 37.06 & 33.12 & $3.329^{*}$ \\
\hline $\begin{array}{l}\text { Edad de los } \\
\text { niños y niñas }\end{array}$ & 94.00 & 75.00 & 71.23 & $3.149^{*}$ \\
en el momento & & & & \\
del estudio & & & & \\
$* p<.05$ & & & & \\
& & & & \\
\end{tabular}

\section{Adaptación inicial y convivencia actual}

$\mathrm{Al}$ analizar la adaptación inicial de los tres grupos encontramos mayores conflictos también en los primeros momentos de la convivencia por las Familias $A$, tanto en la valoración respecto a su adaptación inicial general $(p<.05)$ como respecto a la adaptación inicial a la madre $(p<.05)$, a la motivación escolar inicial $(p<.01)$ y al desempeño escolar inicial $(p<.01)$.

Respecto al nivel de desarrollo a la llegada a nuestro país (Battelle), entre los niños y niñas del grupo de Familias $A$ se encontraba una mayor proporción de niños y niñas con graves retrasos en el desarrollo a la llegada (77\%), en comparación con el porcentaje de estos niños entre las Familias $B$ $(35 \%)$ y las Familias $C(47 \%)\left(\chi^{2}=7.950, p<.05\right)$.

\section{Ajuste psicológico de los niños y niñas y convivencia familiar}

Por último, comparamos las puntuaciones en CI en el momento del estudio (Battelle y WISC-r) entre los chicos de los tres grupos de familias (Tabla 6). Aunque el grupo de
Familias $A$ presenta la puntuación media más baja, el ANOVA muestra que las diferencias no son significativas $(F(2$, $\left.145)=2.077, p^{>} .05\right)$. Si tomamos la variable CI como cualitativa diferenciando entre los que presentan problemas graves (por debajo de un CI de 77 puntos) del resto, existen diferencias entre los tres clusters $\left(\chi^{2}=7.870, p<.05\right)$. Las Familias $A$ están sobrerrepresentadas entre los chicos y chicas con mayores problemas de desarrollo en el momento del estudio, mientras que las $C$ representan un porcentaje mayor entre los chicos y chicas sin problemas de desarrollo.

Tabla 6. Tabla de contingencia. Distribución de clusters entre niveles de CI.

\begin{tabular}{llllll}
\hline & & Familias A & Familias & Familias & Total \\
\hline $\mathrm{CI}<77$ & $\mathrm{~N}$ & 6 & 14 & 11 & 31 \\
& $\%$ del cluster & $50.0 \%$ & $22.6 \%$ & $14.9 \%$ & $20.9 \%$ \\
& Residuos corregidos 2.6 & .4 & -1.8 & \\
$\mathrm{CI}>76$ & $\mathrm{~N}$ & 6 & 48 & 63 & 117 \\
& $\%$ del cluster & $50.0 \%$ & $77.4 \%$ & $85.1 \%$ & $79.1 \%$ \\
& Residuos corregidos & -2.6 & -.4 & 1.8 & \\
\hline
\end{tabular}

\section{Discusión}

La primera conclusión a extraer de los datos que presentamos es que las familias valoran muy en positivo los primeros momentos de la convivencia con sus hijos, presentándonos una adaptación inicial fácil o sin especiales dificultades. Y esto es así pese a que estamos hablando de un grupo de niños y niñas que no eran recién nacidos, recordemos que la media de edad a su llegada era de 3 años, provenientes, a excepción de los de Colombia y Guatemala, de países con una lengua distinta a la española. Hay que tener en cuenta, además, que en las adopciones internacionales el viaje de las familias a los países de sus hijos, o la propia adaptación cultural de los niños, supone un potencial de dificultad añadida a la adaptación. Las familias nos hablan de una muy buena adaptación tanto a las personas como a las costumbres; e incluso cuando valoran una adaptación menos positiva, como es el caso de la escuela, las dificultades se refieren al ámbito académico más que al de las relaciones interpersonales. Esta valoración positiva de la convivencia inicial ha sido mostrada también por otros estudios con muestras de adopción internacional en nuestro país (Berástegui, 2007; Fuentes-Peláez, 2009; Loizaga et al., 2009). Desgraciadamente, no tenemos mucha información respecto a la dinámica familiar en los primeros momentos de la convivencia que pudieran completar esta percepción en principio positiva de las familias. Estudios como el de van Londen, Juffer y van Ijzendoorn (2007) muestran que la mayoría de los niños de adopción internacional establecen apegos seguros con sus padres al poco tiempo de la adopción y funcionan con niveles de desarrollo normativos. Y esta buena adaptación inicial parece ser generalizada, incluso con niños y niñas que llegan con problemas de desarrollo. Esto no quiere decir que la convivencia inicial en estas familias haya estado exenta de dificultades, especialmente para las familias que adoptaron niños y niñas con problemas de desarrollo, cuyos hijos en el momento del estudio manifiestan más problemas de comportamiento. Estos 
datos son especialmente destacables si tenemos en cuenta que esta etapa inicial en el ciclo vital de la familia ha sido considerada crítica para el futuro ajuste de la adopción (Berástegui, 2003; Fuentes-Peláez, 2006). La intervención familiar previa a la adopción puede ser una de las claves para facilitar esta adaptación inicial, en la medida que prepare a las familias para la transición a su paternidad adoptiva, previendo y trabajando sobre las herramientas útiles ante los retos que encontrarán (León et al., 2010).

En segundo lugar, y en consonancia con otros estudios que han abordado el tema (Fuentes et al., 2003; Palacios y Sánchez-Sandoval, 1996), los datos que aquí presentamos nos hablan de las estrechas relaciones entre los problemas manifestados por los niños y niñas adoptados y otras dimensiones implicadas en la dinámica familiar. Al analizar la adaptación de los niños pasados unos años de convivencia, las correlaciones nos indican, por ejemplo, que cuando los estilos educativos paternos se acercan más a los patrones democráticos los comportamientos de sus hijos son también más cercanos emocionalmente y menos conflictivos. Nos referimos únicamente a las relaciones halladas, ya que no es posible inferir relaciones causales entre estos dos tópicos, pues con los datos y análisis realizados es imposible identificar la dirección de las relaciones, es decir, si es una dinámica familiar menos eficaz la que causa problemas en el comportamiento de estos chicos, o si son los mayores problemas manifestados por los chicos los que complican las relaciones familiares. Estos datos pueden entenderse mejor cuando se contempla a la familia desde una perspectiva ecológica y sistémica (Minuchin, 1985; Rodrigo y Palacios, 1999), que insiste en la bidireccionalidad de influencias y relaciones en las dinámicas establecidas entre padres e hijos.

Conociendo estas relaciones, hemos optado por seguir profundizando en los análisis tomando a la familia como sistema, formando clusters o grupos de familias en función de las medidas procedentes de padres e hijos. Así, hemos encontrado un pequeño grupo de familias en el que las cosas parecen estar resultando más complicadas que en el resto, Familias $A$, en el sentido de que los niños y niñas presentan más problemas de comportamiento y más frialdad emocional hacia sus padres, y una mayor proporción de niños con un cociente intelectual bajo (por debajo del percentil 77). Al mismo tiempo, los padres son menos afectuosos y comunicativos, percibiendo en su familia menor cohesión y adaptabilidad que en las otras familias. Lo interesante de este análisis es que nos hemos centrado en la dinámica familiar cuando habían pasado años desde la adopción. Sabemos que niños y niñas adoptados internacionalmente con graves problemas de desarrollo tienen mayor probabilidad de seguir presentando años después mayores problemas de comportamiento (Palacios, Sánchez-Sandoval y León, 2005b), pero igualmente sabemos que adecuadas prácticas educativas familiares se relacionan con un sano desarrollo psicológico durante la infancia y la adolescencia (Oliva, Parra y Arranz, 2008). Más allá de aspectos más específicos de la adopción, las familias adoptivas tienen que responder a todas las exi- gencias educativas de cualquier familia. Independientemente de las características iniciales de los niños que se adoptan, la convivencia familiar se ve facilitada cuando el contexto familiar permite cierta flexibilidad y se adapta a la llegada de un nuevo miembro, así como cuando las estrategias educativas se fundamentan en altas dosis de afecto, de diálogo, y de control, y responden a las necesidades y características concretas de cada niño o niña. De hecho, una paternidad eficaz, de calidad, es un importante factor moderador respecto a la influencia de las características de riesgo iniciales de los niños adoptados sobre la competencia mostrada por estos niños (Masten, 2001; Kriebel y Wentzel, 2011). Como Jiménez y Muñoz (2005) indican, hoy en día es imposible entender y explicar los procesos socializadores en la familia sin tener en cuenta la contribución conjunta de todos los factores implicados (de los padres, de los niños, situacionales, y extrafamiliares).

En tercer lugar, nuestros datos nos permiten reflexionar sobre la cuestión de si existe relación entre los momentos iniciales del ajuste de estos niños y su convivencia familiar, y la posterior adaptación a sus familias años después de la adopción. Los datos de otros trabajos indican que las posibilidades de recuperación de estos niños y niñas es muy elevada, al tiempo que siguen manteniéndose, aunque en menor proporción, niños con retrasos en el desarrollo (Palacios, Román y Camacho, 2010). Queremos destacar y tener presente que si la realidad actual de parte de estos niños y niñas es más complicada, tal es el caso de las Familias $A$, también lo fueron sus inicios, eran niños y niñas que habían sido adoptados con más edad, con mayores problemas de desarrollo y que tuvieron también una peor adaptación inicial. Es interesante ver cómo el reducido grupo de familias que en la actualidad presenta mayores dificultades, eran familias que ya desde los inicios encontraron una adaptación familiar más complicada por parte de sus hijos. Entendemos que lo que estos datos están identificando es un grupo de adopciones donde el equilibrio entre las necesidades infantiles y familiares de partida, y la capacidad familiar de responder a ellas era insuficiente; un grupo de adopciones que podrían haberse beneficiado de un acompañamiento profesional, especialmente al principio, pero también puntual en distintos momentos posteriores de la convivencia. En los últimos años se han ido desarrollando en nuestro país distintos servicios profesionales postadopción para dar respuesta a esta demanda (ver por ejemplo, Benet, Blanxart, Prats y Rossell, 2007; Forment y Aracil, 2007; García, 2007; Magán y Tarazona, 2007; u Ortíz y Rosso, 2007), y que sin duda serán de gran valor para la adaptación de estos niños y niñas

Entre las limitaciones de este trabajo queremos resaltar que la adaptación inicial sólo ha podido ser medida de manera retrospectiva, a través de la percepción y recuerdo de las propias familias. No fue posible realizarlo de otra manera ya que en el momento de iniciar el estudio los niños y niñas llevaban una media de tres años adoptados por sus familias. Sería deseable revisar y perfeccionar las medidas que evalúan la adaptación inicial, al tiempo que iniciar estudios longitudi- 
nales que permitan medir in situ las peculiaridades y necesidades que estas familias encuentran en los momentos iniciales de la convivencia con sus hijos. Igualmente, y aunque la mayoría presente una adaptación muy positiva tras años de convivencia familiar, para trabajos futuros podría tratarse de identificar qué variables pueden estar interviniendo como mediadoras entre las necesidades especiales encontradas por algunas familias al inicio de la convivencia, que han dificultado la adaptación inicial, y la futura adaptación, ya que sólo

\section{Referencias}

Amorós, P. (1987). La adopción y el acogimiento familiar. Madrid: Narcea.

Arranz, E., Oliva, A., Olabarrieta, F. y Antolín, L. (2010). Análisis comparativo de las nuevas estructuras familiares como contextos potenciadores del desarrollo psicológico infantil. Infancia y Aprendizaje, 33 (4), 503-513.

Benet, C., Blanxart, N., Prats, D. y Rossell, M. (2007). El servicio de atención post-adoptiva en Cataluña. Anuario de Psicología, 38 (2), 273-281.

Berástegui, A. (2003). Las adopciones internacionales truncadas y en riesgo en la Comunidad de Madrid. Madrid: Consejo Económico y Social. Comunidad de Madrid.

Berástegui, A: (2007). La adaptación familiar en adopción internacional: un proceso de estrés y afrontamiento. Anuario de Psicología, 38(2), 209-224.

Bernedo, I.M., Fuentes, M.J. y Fernández, M. (2005) Percepción del grado de conflicto en familias adoptivas y no adoptivas. Psicothema, 17 (3), 370374.

Bernedo, I.M., Fuentes, M.J., Fernández-Molina, M. y Bersabé, R. (2007). Percepción de las estrategias de socialización parentales en familias adoptivas y no adoptivas, Psicothema, 19 (4), 597-602.

Brodzinsky, D. M., Smith, D. W. y Brodzinsky, A. B. (1998). Children's adjustment to adoption. (Vol. 38). London: SAGE.

Forment, C. y Aracil, A. (2007). El acompañamiento profesional a la familia adoptiva. Anuario de Psicología, 38 (2), 253-258.

Fuentes, M.J., Motrico, E. y Bersabé, R. (2003). Estrategias de socialización de los padres y conflictos entre padres e hijos en la adolescencia. Anuario de Psicología, 34(3), 385-400.

Fuentes-Peláez, N. (2006). Elementos de estrés percibidos por las familias adoptivas internacionales durante el ajuste inicial y estrategias utilizadas para afrontarlos. 1er Forum Internacional de Infancia y Familias. Barcelona, 29 de septiembre a 3 de octubre.

Fuentes-Peláez, N. (2009). Adoption internationale en Catalogne (Espagne). Adaptation et processus d'integration familiale. La revue internationale de l'éducation familiale, 25, 37-62.

García, M. (2007). El servicio de atención post-adoptiva en la Comunidad Valenciana. El apoyo a la familia adoptiva. Anuario de Psicología, 38 (2), 247-252.

Hernández-Muela, S., Mulas, F., Téllez de Meneses, M., y Roselló, B. (2003). Niños adoptados: factores de riesgo y problemática neuropsicológica. Revista de Neurología, 36, 108-117.

Hogg, C., Rutter, M. y Richman, N. (1997). Emotional and behavioural problems in children. En I. Sclare (Ed.), Child psychology portfolio. Windsor: NFER-Nelson.

Jiménez, J. y Muñoz, A. (2005). Socialización familiar y estilos educativos a comienzos del siglo XXI . Estudios de Psicología, 26 (3), 315-327.

Johnson, D. E., Miller, L. C., Iverson, S., Thomas, W., Franchino, B., Dole, K., Kiernan, M.T., Georgieff, M.K., y Hostetter, M.K. (1992). The health of children adopted from Romania. Journal of the American Medical Association, 268, 3446-3451.

Juffer, F. y van Ijzendoorn, M.H. (2005) Behavior problems and mental health referrals of international adoptees: A meta-analytic approach, JAMA, Journal of the American Medical Association, 293, 2501-2515.

Kirschner, D. (1990). The adopted child syndrome: Considerations for psychotherapy. Psychotherapy in private practice, 8(3), 93-100. algunas de las que tuvieron difíciles comienzos siguen manifestando dificultades con el tiempo.

Agradecimientos.- Este trabajo ha sido financiado por el Ministerio de Trabajo y Asuntos Sociales de España (Proyecto Los procesos familiares en la adopción y la evolución de los adoptados: análisis de las rupturas, SUBVCON2003-01) y por la Consejería de Sanidad y Bienestar Social de la Junta de Castilla y León (Estudio sobre la adaptación y evolución de los niños adoptados por familias de Castilla y León de adopción internacional, SUBCON2003-002.

Kriebel, D.K. y Wentzel, K. (2011). Parenting as a moderator of cumulative risk for behavioral competence in adopted children. Adoption Quarterly, 14, 37-60.

Lanz, M., Lafrate, R., Rosnati, R. y Scabini, E. (1999). Parent-child communication and adolescent self-esteem in separated, intercountry adoptive and intact non-adoptive families. Journal of Adolescence, 22(785794).

León, E., Sánchez-Sandoval, Y., Palacios, J. y Román, M. (2010). Programa de formación para la adopción en Andalucía. Papeles del Psicólogo, 31(1), 202-210.

Levy-Shiff, R. (2001). Psychological adjustment of adoptees in adulthood: Family environment and adoption-related correlates. International Journal of Behavioral Development, 25(2), 97-104.

Levy-Shiff, R., Bar, O., y Har Even, D. (1990). Psychological adjustment of adoptive parents-to-be. American Journal of Orthopsychiatry, 60, 258-267.

Levy-Shiff, R., Goldsmith, I. y Har-even, D. (1991). Transition to Parenthood in Adoptive Families. Developmental Psychology, 27(1), 131- 140.

Levy-Shiff, R., Zoran, N. y Shulman, S. (1997). International and Domestic Adoption: Child, Parents, and Family Adjustment. International Journal of Behavioral Development, 20(1), 109-129.

Loizaga, F., Louzao, F., Aranzabal, M. y Labayru, M. (2009). Adopción internacional: ¿Cómo evolucionan los niños, las niñas y sus familias?. Bilbao: Universidad de Deusto.

López, C. y García, A. (1997). Variables relevantes en el acogimiento familiar preadoptivo: Un estudio empírico en la Región de Murcia. Revista AMAIN, 97, 1-5.

Magán, M. y Tarazona, M.C. (2007). El trabajo social con la família adoptiva. Anuario de Psicología, 38 (2), 259-264.

Marcovitch, S., Goldberg, S., Gold, A., Washington, J., Wasson, C., Krekewich, K. y Handley-Derry , M. (1997). Determinants of Behavioural Problems in Romanian Children Adopted in Ontario. International Journal of Behavioral Development, Vol. 20, 1, 17-31.

Masten, A.S. (2001). Ordinary magic: Resilience processes in development. American Psychologist, 56(3), 227-238.

Minuchin, P. (1985). Families and individual development: provocations from the field of family therapy. Child Development, 56, 289-302.

Molina, M. (2002). Descripción del proceso de adaptación infantil en adopciones especiales. Dificultades y cambios observados por los padres adoptivos. Anales de Psicología, 18(1), 151-168.

Newborg, J., Stock, J.R., y Wnek, L. (1988): Battelle: developmental inventory. Allen, Texas: DLM (trad. Cast.: Battelle: Inventario de desarrollo. Madrid: TEA, 2001).

O'Connor, T. G., Rutter, M., Beckett, C., Keaveney, L., Kreppner, J. M., and the English and Romanian Adoption Adoptees Study Team (2000). The effects of global severe privation on cognitive competence: Extension and longitudinal follow-up. Child Development, 71(2), 376-390.

Oliva, A., Parra, A. y Arranz, E. (2008). Estilos relacionales parentales y ajuste adolescente. Infancia y Aprendizaje, 31, 93-106.

Olson, D. H., Portner, J. y Bell, R. Q. (1982). FACES II: Family adaptability and cohesion evaluation scales . St. Paul, Minnesota: Family Social Science. University of Minnesota.

Ortíz, P. y Rosso, D. (2007). El servicio de atención post-adoptiva en Andalucía. Anuario de Psicología, 38 (2), 265-272. 
Palacios, J. y Amorós, P. (2006). Recent Changes in Adoption and Fostering in Spain. British Journal of Social Work, 36, 921-935.

Palacios, J., Román, M. y Camacho, C. (2010). Growth and Development in Internationally Adopted Children: Extent and Timing of Recovery After Early Adversity. Child: Care, Health and Development, 1-7.

Palacios, J. y Sánchez-Sandoval, Y. (1996). Relaciones padres-hijos en familias adoptivas. Anuario de Psicología, 71, 87-105.

Palacios, J. y Sánchez-Sandoval, Y. (1998). Tipología de familias adoptivas. Bienestar y protección infantil, IV, 1, 7-26.

Palacios, J. y Sánchez-Sandoval, Y. (2000). Escala Revisada de Evaluación de Estilos Educativos (4er). Universidad de Sevilla.

Palacios, J., Sánchez-Sandoval, Y. y León, E. (2005a) Adopción Internacional en España: un Nuevo País, una Nueva Vida. Madrid. Ministerio de Trabajo y Asuntos Sociales.

Palacios, J., Sánchez-Sandoval, Y. y León, E. (2005b). Adopción y problemas de conducta. Revista Internacional de Diagnóstico y Evaluación Psicológica, 19(1), 171-190.

Quinton, D., Rushton, A., Dance, C. y Mayes, D. (1998). Joining New Families. West Sussex: Wiley.

Rodrigo, M. J. y Palacios, J. (1999). Conceptos y dimensiones en el análisis evolutivo-educativo de la familia. En M. J. Rodrigo y J. Palacios (Coords.), Familia y desarrollo bumano (pp. 45-70). Madrid: Alianza.

Rosnati, R. y Marta, E. (1997). Parent-child relationships as a protective factor in preventing adolescents' psychological risk in inter-racial adoptive and non-adoptive families. Journal of Adolescence, 20, 617-631.

Rueter, M.A. y Koerner, A.F. (2008). The effect of family communication patterns on adopted adolescent adjustment. Journal of Marriage and Family, 70, 715-727.
Rutter, M.L., Kreppner, J.M. y O'Connor, T.G. (2001). Specificity and heterogeneity in children's responses to profound institutional privation. British Journal of Psychiatry, 179, (2), 97-103.

Sánchez-Sandoval, Y. (2002). El ajuste de niños y niñas adoptados y su vida familiar: un estudio longitudinal. Sevilla: Tesis doctoral no publicada.

Sánchez-Sandoval, Y. y Palacios, J. (2004). La escala revisada de evaluación de estilos educativos (4er): análisis psicométrico tras su aplicación en un grupo de familias adoptivas. Póster presentado en the VII European Conference on Psychological Assessment. Málaga, 1 a 4 de abril.

Sánchez-Sandoval, Y., Palacios, J. y León, E. (2002): Entrevista sobre el proceso de adopción internacional (EPAI). Documento no publicado, Universidad de Sevilla.

Sánchez-Sandoval, Y., Palacios, J. y León, E. (2004). Características de los Niños y Niñas Procedentes de Adopciones Internacionales. Portularia: Revista de Trabajo Social. Vol. 4, 269-276.

Schaefer, E. S. y Edgerton, M. D. (1977): Parent Report of Child Behavior to the Parent: University of North Carolina, Chapel Hill.

Van Londen, W.M., Juffer, F. y van Ijzendoorn, M.H. (2007). Attachment, Cognitive, and Motor Development in Adopted Children: Short-term Outcomes after International Adoption. Journal of Pediatric Psychology, 32(10), 1249-1258.

Weschler, D. (1974): Weschler scale for children revised. Nueva York: The Psychological Corporation (trad. cast.: Escala de inteligencia de Weschler para niños revisada. Madrid: TEA, 2001).

(Artículo recibido: 16-05-2011, revisión: 13-10-2011, aceptado: 13-10-2011) 